\title{
A Masing Molecular Cloud in the Central Parsecs of Mrk 348
}

\author{
Peck, A. B. ${ }^{1}$, Henkel, C. ${ }^{2}$, and Ulvestad, J. S. ${ }^{3}$ \\ 1 Harvard Smithsonian CfA, SMA Project, P.O. Box 824, Hilo, HI 96720 USA \\ apeck@cfa.harvard.edu \\ 2 Max-Planck-Institut für Radioastronomie, Auf dem Hügel 69, 53121 Bonn, \\ Germany p220hen@mpifr-bonn.mpg.de \\ 3 NRAO, P.O. Box 0, Socorro, NM 87801 USA julvesta@nrao.edu
}

We report new observations of the $\mathrm{H}_{2} \mathrm{O}$ megamaser in the Seyfert 2 galaxy Mrk 348. The line is redshifted by $\sim 130 \mathrm{~km} \mathrm{~s}^{-1}$ with respect to the systemic velocity, is extremely broad, with a FWHM of $130 \mathrm{~km} \mathrm{~s}^{-1}$, and has no detectable high velocity components within $1500 \mathrm{~km} \mathrm{~s}^{-1}$ on either side of the observed line. The unusual line profile led us to suspect that this source, like NGC 1052 [1], might belong to a class of megamaser galaxies in which the amplified emission is the result of an interaction between the radio jet and an encroaching molecular cloud, rather than occurring in a circumnuclear disk. Our initial VLBA observations show that the maser emission emanates entirely from a region $\leq 0.25 \mathrm{pc}$ in extent, located toward a continuum component thought to be associated with the receding jet [6]. The very high linewidth occurring on such small spatial scales and the rapid variability indicate that the $\mathrm{H}_{2} \mathrm{O}$ emission is more likely to arise from a shocked region at the interface between the energetic jet material and the molecular gas in the cloud where the jet is boring through, than simply as the result of amplification by molecular clouds along the line of sight to the continuum jet. The orientation of the radio jets close to the plane of the sky also results in shocks with the preferred orientation for strong masers from our vantage point. Singledish monitoring with the Effelsberg $100 \mathrm{~m}$ telescope showed that the line and continuum emission "flared" on very similar timescales. The close temporal correlation between this activity in the maser emission and the continuum flare further suggest that the masing region and the continuum hotspots are nearly equidistant from the central engine and may be different manifestations of the same dynamical events. The study of this newly discovered type of $\mathrm{H}_{2} \mathrm{O}$ megamaser can provide detailed information about the conditions in the ISM in the central 1-10 pc of active galactic nuclei.

\section{Introduction}

$\mathrm{H}_{2} \mathrm{O}$ megamasers are best known as a means to probe the accretion disks in Seyfert galaxies. In the most famous source, NGC 4258, a thin, slightly warped, nearly edge-on disk orbits in Keplerian rotation around a central 
mass of $4 \times 10^{7} \mathrm{M}_{\odot}$ (e.g. [5]). VLBI studies have been used to determine the size and shape of this warped molecular disk as traced by the maser spots. A few other sources show evidence of a toroidal structure, but the distribution of maser spots is not as well understood. There is now evidence, however, for a distinct class of $\mathrm{H}_{2} \mathrm{O}$ megamaser. In these sources the amplified emission is the result of an interaction between the radio jet and an encroaching molecular cloud, rather than occurring in a circumnuclear disk. The only known sources in this class were NGC 1068 [3] and the Circinus galaxy [4], which appear to have both a circumnuclear disk and maser emission arising along the edges of an ionization cone or outflow, and NGC 1052, in which the masers appear to arise along the jet and have a full width at half maximum (FWHM) $\sim 90$ $\mathrm{km} \mathrm{s}^{-1}$ [1]. We have recently identified the fourth such source, Mrk 348, a Seyfert 2 galaxy with a low inclination angle and an exceptionally bright and highly variable nuclear radio source [2]. Observations of Mrk 348 using Global VLBI at $4.8 \mathrm{GHz}$ [7] show two faint continuum hotspots on either side of a much brighter central peak. The relative intensities of the two outer components are quite similar, indicating that relativistic beaming effects are probably minimal and the jet axis should be close to the plane of the sky. Ground-based observations [8] show evidence of an ionization cone with a half-angle of $\sim 45^{\circ}$, which also suggests a jet axis fairly close to the plane of the sky.

The initial detection of the flaring maser in Mrk 348 using the Effelsberg 100m telescope took place in 2000 March [2]. The $\mathrm{H}_{2} \mathrm{O}$ maser line in Mrk 348 is extremely broad, with a FWHM of $\sim 130 \mathrm{~km} \mathrm{~s}^{-1}$, though in many of the monitoring epochs the emission appears to consist of 2 lines which can be tentatively fit with a broad Gaussian function at $\sim 4609 \mathrm{~km} \mathrm{~s}^{-1}$ with FWHM $\sim 100 \mathrm{~km} \mathrm{~s}^{-1}$ and a narrower one at $\sim 4678 \mathrm{~km} \mathrm{~s}^{-1}$ with FWHM $\sim 60$ $\mathrm{km} \mathrm{s}^{-1}$. The amplitudes of each component vary significantly on very short timescales. There are no detectable high velocity components within $1500 \mathrm{~km}$ $\mathrm{s}^{-1}$ on either side of the strong emission line [2].

Figure 1a shows 5 spectra which were chosen to emphasize the significant variations in the line profiles over our first 16 months of observations. The first profile is the discovery spectrum, where both components had a flux of around $30 \mathrm{mJy}$. In the second profile, taken within a week of our VLBA observations, both components verged on undetectability and then, in the third profile, the higher velocity component flared to twice the intensity of the year previous, and the lower velocity component disappeared altogether. This change was reversed in June, shown in the fourth profile, when the lower velocity component was present, though the intensity was low, and the higher velocity component vanished. Within two weeks, both components were once again nearly equal. Figure 1b shows the variation in line and continuum flux density with respect to time. Comparison shows that the continuum flux varies loosely with the maser flux. The close temporal correlation between the flaring activity in the maser emission and the continuum flare suggest that 

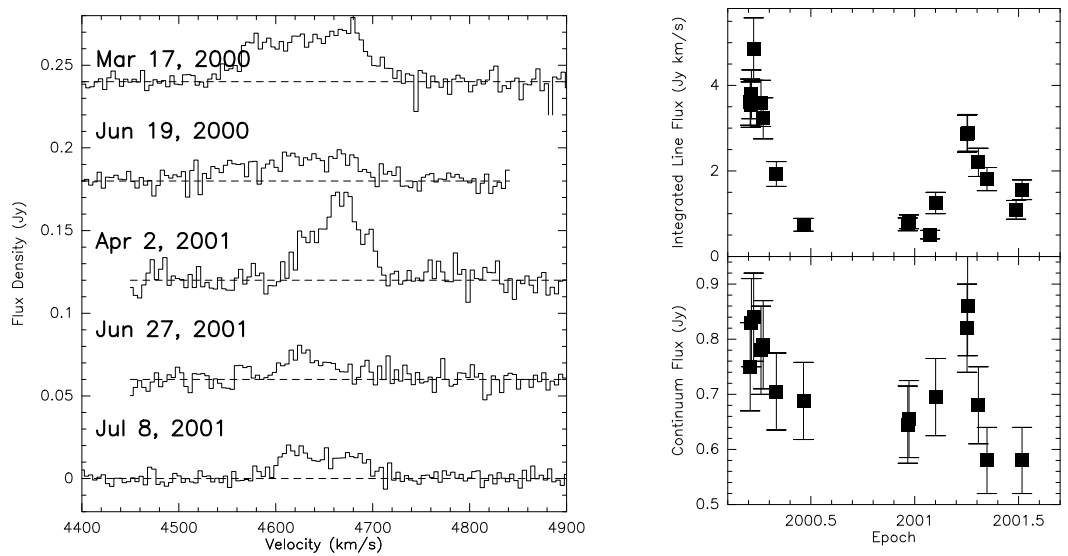

Fig. 1. Left) $5 \mathrm{H}_{2} \mathrm{O}$ spectra from the Effelsberg 100-m telescope chosen to illustrate the variations in the profiles seen on timescales of days or months. Top right) Integrated line flux density vs. time; Bottom right) continuum flux density vs. time measured with the Effelsberg 100-m telescope. The variations in line and continuum intensity can be seen to vary concurrently.

the masing region and the continuum hotspots are nearly equidistant from the central engine and may be different manifestations of the same dynamical events. VLBA continuum observations indicate that the center of activity in Mrk 348 is likely to be located between the two strongest central components [6]. We hypothesize that at an epoch prior to 1998.75 , jet components were ejected simultaneously in the approaching and receding jets, leading to both the continuum flare and the new flaring activity of the maser.

Figure 2a shows three integrated line profiles toward Mrk 348 taken using the VLBA. The maser emission is clearly seen to lie along the line of sight to the fainter northern continuum component, rather than the brightest region of the continuum source which is thought to contain the core. The Gaussian fit to the line shown in the upper left profile has an amplitude of $14 \pm 2 \mathrm{mJy}$ and an integrated flux of $2.11 \pm 0.34 \mathrm{Jy} / \mathrm{beam} / \mathrm{km} \mathrm{s}^{-1}$, indicating that all of the flux measured in the Effelsberg May 2 observation has been recovered. The FWHM is $139 \pm 11 \mathrm{~km} \mathrm{~s}^{-1}$ centered on $4641 \pm 2.2 \mathrm{~km} \mathrm{~s}^{-1}$, consistent with the single-dish measurements and redshifted by $134 \mathrm{~km} \mathrm{~s}^{-1}$ with respect to the systemic velocity.

In order to produce the maser emission detected in this source, the gas in the molecular cloud within the central parsecs of Mrk 348 must have a pre-shock density ranging from around $10^{6} \mathrm{~cm}^{-3}$ to a few times $10^{9} \mathrm{~cm}^{-3}$. An expanding bow-shock being driven into this cloud by the AGN jet has a velocity between $135 \mathrm{~km} \mathrm{~s}^{-1}$ and $0.5 \mathrm{c}$ in the direction of jet propagation, and between $35 \mathrm{~km} \mathrm{~s}^{-1}$ and $300 \mathrm{~km} \mathrm{~s}^{-1}$ at various points along the oblique edges (Figure 2b). This shock generates a region of very high temperature, 

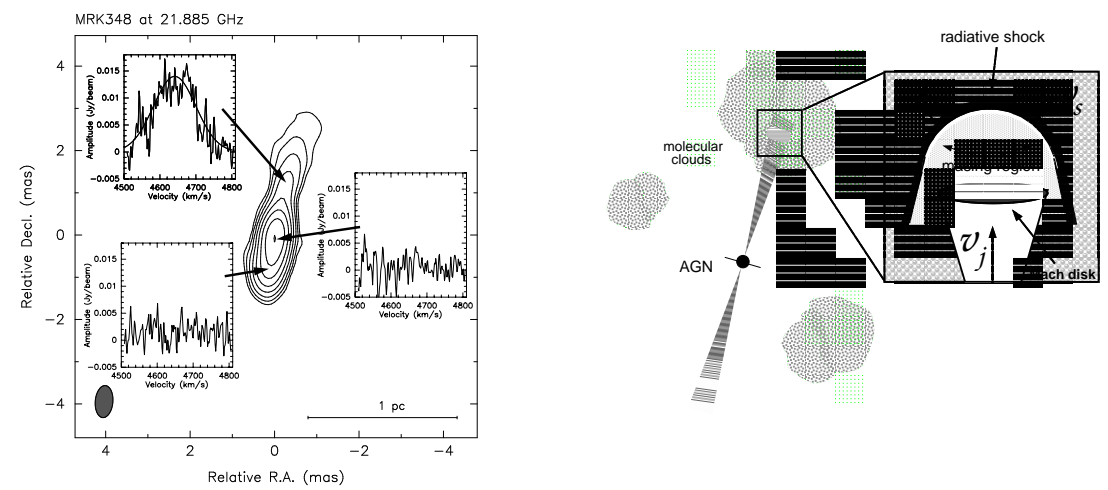

Fig. 2. Left) $\mathrm{H}_{2} \mathrm{O}$ line profiles toward Mrk 348, superimposed on the continuum map made from 20 line-free channels extracted from the low frequency end of the observed frequency range. The RMS noise in the line profiles is $\sim 4 \mathrm{mJy}_{\text {beam }}^{-1}$ channel $^{-1}$. The continuum image is naturally weighted and the lowest contour is $5 \mathrm{mJy}$. The RMS noise in the continuum map is less than $1 \mathrm{mJy}^{\text {beam }^{-1}}$. Right)A cartoon model of the expanding bubble caused by the jet material impacting the molecular cloud. The maser emission arises within the region surrounded by the radiative shock.

$\left(\leq 10^{5} \mathrm{~K}\right)$, which dissociates the molecular gas and to some extent shatters the dust grains expected to be present and/or evaporates their icy mantles. Immediately following this shock, $\mathrm{H}_{2}$ begins forming on the surviving dust grains when the temperature has dropped to $\sim 1000 \mathrm{~K}$, and this in turn provides sufficient heating to stabilize the temperature at $\sim 400 \mathrm{~K}$, with gas densities of $\sim 10^{8} \mathrm{~cm}^{-3}$ and ultimately an $\mathrm{H}_{2} \mathrm{O}$ abundance as high as $10^{-5}$. Ammonia is not found because the levels of UV radiation remain too high, but the detection of other molecular species will help to test this scenario.

\section{References}

1. Claussen, M. J., Diamond, P. J., Braatz, J. A., et. al. 1998, ApJL, 500, 129

2. Falcke, H., Henkel, C., Peck, A. B., et. al. 2000, A\&A, 358, L17

3. Gallimore, J. F., Baum, S. A., O'Dea, C. P., et. al. 1996, ApJ, 462, 740

4. Greenhill, L. J., Moran, J. M., Booth, R. S., et. al 2001, in IAU Symposium 205 eds. R. Schilizzi, S. Vogel, F. Paresce, M. Elvis, 334

5. Miyoshi, M., Moran, J., Herrnstein, J., et. al. 1995, Nature 373, 127

6. Peck, A. B., Henkel, C., Ulvestad, J. S., et. al., 2003, ApJ, 590, 149

7. Roy A. L., Ulvestad J. S., Wilson A. S., et. al. 1999, in Perspectives on Radio Astronomy: Science with Large Antenna Arrays, [Dwingeloo: NFRA], Ed. M.P. van Haarlem, p. 173

8. Simpson, C., Mulchaey, J. S., Wilson, A. S., et. al. 1996, ApJ, 457, L19 